\title{
Symbolic Security Analysis of Ruby-on-Rails Web Applications
}

\author{
Avik Chaudhuri Jeffrey S. Foster \\ University of Maryland, College Park \\ \{avik, jfoster\}@cs. umd.edu
}

\begin{abstract}
Many of today's web applications are built on frameworks that include sophisticated defenses against malicious adversaries. However, mistakes in the way developers deploy those defenses could leave applications open to attack. To address this issue, we introduce Rubyx, a symbolic executor that we use to analyze Ruby-onRails web applications for security vulnerabilities. Rubyx specifications can easily be adapted to a variety of properties, since they are built from general assertions, assumptions, and object invariants. We show how to write Rubyx specifications to detect susceptibility to cross-site scripting and cross-site request forgery, insufficient authentication, leaks of secret information, insufficient access control, as well as application-specific security properties. We used Rubyx to check seven web applications from various sources against our specifications. We found many vulnerabilities, and each application was subject to at least one critical attack. Encouragingly, we also found that it was relatively easy to fix most vulnerabilities, and that Rubyx showed the absence of attacks after our fixes. Our results suggest that Rubyx is a promising new way to analyze Ruby-on-Rails web applications for security vulnerabilities.
\end{abstract}

\section{Introduction}

Today, online services are a crucial part of many industries such as banking, government, healthcare, and retail. Unfortunately, the web applications that underlie these services often face serious security threats, and vulnerabilities in these applications can lead to loss of revenue, damage to credibility, and legal liability.

Many web applications are built on top of frameworks whose APIs provide extensive defense mechanisms against common attacks such as cross-site scripting (XSS) and cross-site request forgery (CSRF). However, the mere existence of these APIs is insufficient-to be effective they must be used correctly by the programmer, who must ensure that the logic of the web application cooperates with the design of the APIs. Moreover, even if security-relevant APIs are used correctly, application-specific security vulnerabilities, such as insufficient access control checks or leaks of confidential information, could still remain.

In this paper, we propose addressing this challenge by using symbolic execution $[16,17,39,40]$ to analyze Ruby-on-Rails (or just "Rails") web applications. Rails is a popular framework based on Ruby, an object-oriented scripting language [1]. We focus on analyzing server-side code, and we are concerned with protecting the web application and honest users from dishonest users or other adversarial clients.

We developed Rubyx, a symbolic executor for Rails, and use it to detect potential vulnerabilities such as XSS, CSRF, susceptibility to session manipulation, and allowing unauthorized access, among others. Unlike most previous work on web-application security $[9,14,24,37,62]$, we do not study such threats in isolation; by using symbolic execution, we can perform end-to-end reasoning about all of these vulnerabilities simultaneously. And although the low-level details of our approach are targeted toward Rails, we believe the same ideas can be applied to many other web application frameworks, such as Merb, CodeIgnitor, Django, and ColdFusion on Wheels.

Briefly, symbolic execution involves running code with symbolic variables, which are unknowns that range over sets of concrete values. At conditional branches involving symbolic variables, the symbolic executor consults an underlying Satisfiability Modulo Theory (SMT) solver to decide which branches could be taken. If both are possible, the executor conceptually forks execution, trying both paths. Thus, if run to completion, symbolic execution explores all paths and hence can verify the absence of vulnerabilities.

Of course, verifying all paths in general would be intractable, since programs can have an unbounded number of paths. However, we have found that web applications are typically "broad" and "shallow"-while there are many possible requests and responses, each request-response path is usually short. Hence this domain is ideal for symbolic execution, because the shallowness of the paths controls the exponential blowup from branches. To handle unbounded data structures, we rely on the small model hypothesis-we initialize arrays with a small number of symbolic variables, and prove the absence of vulnerabilities up to that bound.

A major advantage of our approach is that it is programmable: it can be used to specify and check arbitrary properties of interest. In Rubyx, the programmer calls assert $e$ to check that the (arbitrary) Ruby expression $e$ always evaluates to true; assume $e$ to tell Rubyx to assume that $e$ holds; and defines methods named invariant to specify properties that must be invariant during execution.

Using this simple interface, we show how to encode a variety of security properties at various levels of abstraction. We implemented a proxy Rails API that simulates the original API, and uses notions such as principals, secrecy, and trust to assert XSS safety, CSRF protection, and password authentication. At a high level, XSS safety specifies that only trusted (sanitized) strings can be part of a response; CSRF safety specifies that the principal that sends a request must be at least as trusted as the principal that receives the response; and password authentication specifies that senders and receivers of requests are at least as trusted as the logged-in user. These specifications concisely rule out several classic attacks as well as recent variants studied in the literature [9]. Moreover, our specifications are generic: we can check for XSS safety, CSRF protection, and password authentication simply by symbolically executing a target application in conjunction with our proxy API.

In addition to these generic security specifications, we can also use Rubyx to specify and check application-specific security properties, such as access control and functional correctness. We believe that the breadth of these properties, along with the generic properties above, demonstrates the flexibility and power of using Rubyx to reason about security vulnerabilities.

Rubyx is implemented on top of DRails, a tool we developed previously to "compile" Rails code by making many implicit Rails conventions explicit, which simplifies analysis [3]. Rubyx uses 
Yices [58] as its SMT solver. To improve performance, Rubyx uses several optimizations, including a careful encoding of the necessary constraints in Yices as well as caching to reduce solver queries.

We applied Rubyx to analyze security of seven Rails applications obtained from various sources. Rubyx found several serious vulnerabilities in these applications, including XSS, CSRF, authentication failures, insufficient access control, and applicationspecific problems. Encouragingly, we found that it was generally easy to manually fix these vulnerabilities, and that after doing so Rubyx could show that the attacks were eliminated for the fixed applications. Rubyx took between half a minute to 3 minutes in its analysis of these programs, which range from $5 \mathrm{k}-20 \mathrm{k}$ lines of code. Informally, we found the effort required to apply Rubyx to be similar to what we would expect in testing, and we believe this approach will prove viable in practice. Finally, our experiments revealed several common misunderstandings about the defense mechanisms provided by Rails. We have reported these observations to the Rails security team, and are working with them on improving the design and documentation of these mechanisms.

In summary, this paper makes the following contributions:

- We study a range of attacks and defenses in Rails, and explain the intricacies of correctly using important Rails security APIs. We believe this discussion is of independent interest, as our experiments indicate that developers often do not appreciate the subtleties of Rails's defenses, often rendering them ineffective (Section 2).

- We introduce Rubyx, which we believe is the first symbolic execution engine for Ruby and for Rails. The broad and shallow nature of Rails applications makes them a particularly attractive target for symbolic execution. Rubyx includes several optimizations, including SMT solver query caching (Section 3).

- We show how to encode specifications for XSS, CSRF, password authentication, secrecy, and several application-specific properties using Rubyx's assume/assert annotation mechanism. We believe that these concise formal specifications are not only useful to Rubyx, but that they help clarify the relevant security concerns, and can serve as a guideline for Rails developers (Section 4).

- We evaluate Rubyx and our specifications on seven Rails applications. We discovered several serious attacks against these applications, and that the vulnerabilities were generally straightforward to fix (Section 5). We are working with the Rails security team to ensure that such vulnerabilities can be more easily avoided by future developers.

We believe these results suggest that symbolic execution in general, and Rubyx in particular, is a promising approach for detecting and preventing security vulnerabilities in web applications.

\section{Attacks and Defenses in Rails}

In this section we discuss several important vulnerabilities that can arise in Rails programs. In Section 4, we will see how to detect these vulnerabilities using Rubyx.

For illustration, we use examples from pubmgr, an application developed by one of the authors to manage publications by members of our research group (PLUM ${ }^{1}$ ). Specifically, pubmgr maintains a database of users, authors, and publications. An author must be a member of PLUM or a co-author of some member of PLUM; each author may be linked to several publications. Conversely, a publication may be linked to several authors, some of which must be members of PLUM. A distinguished user, admin, may identify

\footnotetext{
${ }^{1}$ Programming Languages at $\underline{\text { University }}$ of $\underline{\text { Maryland }}$
}

other users as members of PLUM. Such users can then manage their co-authors and publications.

Like all Rails applications, pubmgr consists of three kinds of components: models, which are Ruby class that interface to the database; views, which are either HTML pages with embedded Ruby code or, equivalently, Ruby methods that generate HTML pages; and controllers, which are methods that are invoked when the client requests a web page. A controller receives as inputs any GET or POST parameters submitted by the user, as well as state information encoded in the session. As a controller runs, it may redirect to other controllers, use models to access the database, and return by specifying which view should be rendered in response to the user's request.

\subsection{XSS}

Several web attacks use cross-site scripting (XSS) to execute arbitrary (malicious) code on the browser. In XSS attacks, an adversary embeds executable code (likely JavaScript) in text fields in the web application's database. When a user receives a web page containing those compromised fields, the browser executes the code, possibly leaking the user's secrets or carrying out operations with the user's privileges on behalf of the adversary.

To illustrate potential attacks, consider the following code:

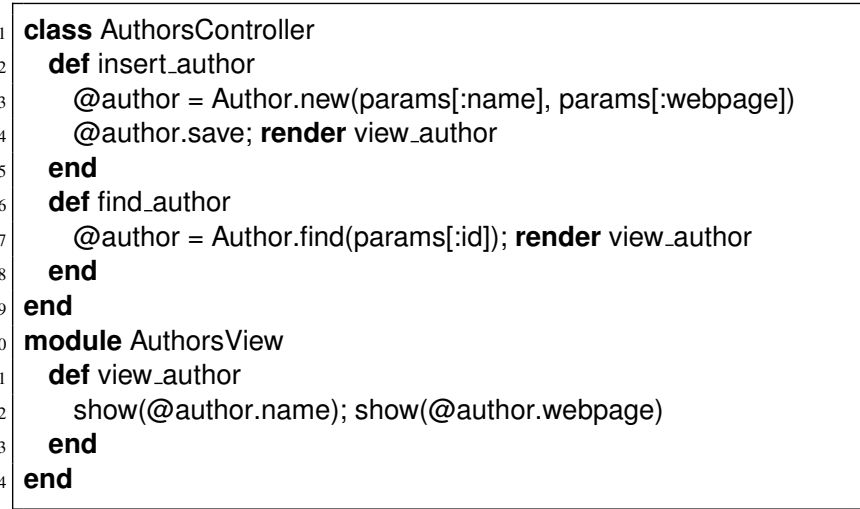

This code contains two controllers. The first, insert_author, is given a name and a web page, which are passed in via the params hash. On line 3, the controller creates a new Author, which is a model representing a database row. The controller then writes the new author to the database and calls view_author to display a web page in response. That view shows the author's name and web page (line 12). Along the same lines, the find_author controller looks up the input author id in the database and renders the same view to show the author's information.

Unfortunately, while this code is straightforward, it is also vulnerable to XSS attacks: an attacker can use insert_author to create an author whose name or web page contains malicious code.

A typical countermeasure against XSS is to sanitize any text that may ultimately be rendered by the browser, to ensure that such inputs do not embed executable code. One way to do this in Rails is to validate text before writing it to the database, as in the code:

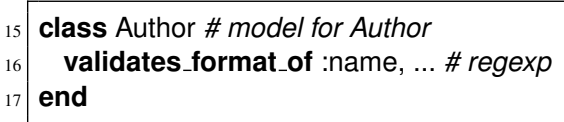

Here the programmer calls the validates_format_of method to tell Rails that before the name field can be written to the database, it must match a given regular expression (elided by ....). In this way we can prevent code from being included in author names.

Another countermeasure is to HTML-escape text before display. Here is code to do just that whenever the webpage field is rendered: 


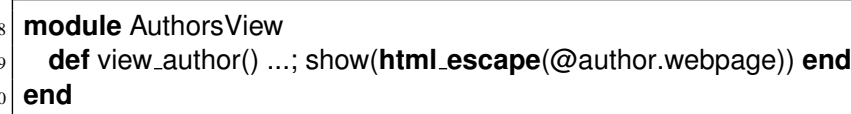

We can see that both of these countermeasures prevent executable code in displayed web pages. Critically, however, the programmer must remember to use them to enable their protection. Moreover, notice that even for something as simple as sanitization there are different approaches, and the point at which sanitization is applied may vary.

\subsection{CSRF}

Recently, cross-site request forgery (CSRF) has emerged as a powerful technique for several web attacks. CSRF has been described by some experts as a "sleeping giant," because its power is (as yet) widely underestimated [33]. CSRF attacks work as follows. Suppose that a user interacts with a web application $A$ while also browsing another web site $B$. Pages retrieved from $B$ may cause the user's browser to send further requests (e.g., GET requests for images) on behalf of the user. By compromising site $B$, an attacker can control those requests; in particular, such requests can be sent to application $A$, and appear to come from the user when in fact they come from the attacker. This is especially harmful if the requests are non-idempotent (i.e., they cause state changes).

Preventing these attacks in Rails requires employing several related countermeasures. First, we ensure that any calls that may change state are POST requests. In the following code, we use Rails's before_filter method to specify that ensure_post must be called before a request is routed to the insert_author controller.

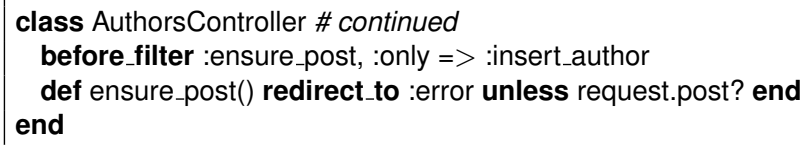

Second, since POST requests can still be surreptitiously issued from other web sites open in the browser [65], we require that POST requests include a secret token, which is only available to web pages that may legitimately send POSTs. We do this by calling Rails's protect_from_forgery method:

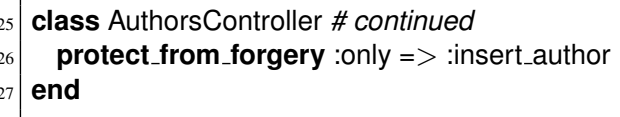

This call ensures that POSTs to insert_author must include a parameter named :authenticity_token (automatically included by Rails in forms), and this parameter must match an internal token returned by form_authenticity_token(), which is part of the Rails API. Here is a fragment of the code we use in Rubyx for this part of the API:

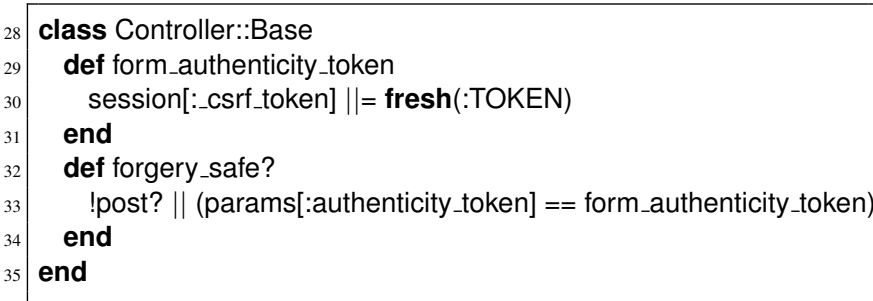

On line 30, form_authenticity_token() either return the current token (stored in session[:_csrf_token]) or generates a fresh one (if session[:_csrf_token] is nil). The method forgery_safe? then ensures that this token matches the relevant parameter for POST requests.

Finally, we must account for "insider attacks," i.e., attacks by users of the application (against other users of the application). To understand this issue, we need to look again at the implementation of token generation on line 30 above. The complication here is that session[:_csrf_token] is not reset automatically by Rails between logins, hence different users that log in from the same IP address could inadvertently be given the same CSRF token. To properly protect against CSRF, the application should always change session[:_csrf_token] to nil before logging in a user, so that the token is regenerated whenever a different user logs in. Rails provides a method, reset_session, which has just this effect:

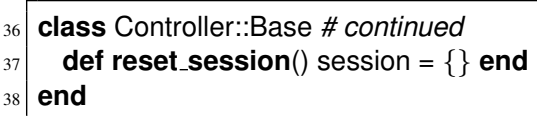

We should stress that this mechanism is fairly delicate; for example, calling reset_session after logging out a user may be inadequate, since we cannot assume that a malicious user will politely log out (and most applications will still log in a different user after).

Our experiments suggest that reset_session is seldom used correctly (if at all) to prevent CSRF attacks. One possible reason is that the Rails documentation for reset_session focuses on XSS attacks, and developers may think it is unnecessary if they take other measures to prevent XSS. In contrast, when we developed a specification for CSRF protection (Section 4), we pinpointed the significance of reset_session for CSRF.

Notice that using CSRF protection is not that easy, and checking that CSRF protection is used correctly requires delicate reasoning. We need to track dynamic checks that ensure requests are POST; we need to distinguish new objects based on context to differentiate tokens generated for different users; and so on. Techniques developed for reasoning about trace properties of security protocols may apply [27] — but such techniques require extensive annotations that the usual Rails developer cannot be expected to provide. In contrast, our symbolic execution-based analysis can readily verify CSRF safety for such code.

\subsection{Session manipulation}

Next, we consider session manipulation attacks. Sessions usually maintain crucial state. For example, after a user successfully authenticates (and logs in), the identity of the user is often stored in the session and trusted by the web application. Furthermore, as we have seen above, CSRF tokens are stored in sessions. Thus, maintaining the integrity of sessions is very important for security.

Rails provides two modes for storing sessions. In the databasestore mode, the session is stored in the database, keyed by an identifier, and the identifier is stored in a cookie. This mode is secure but involves some overhead since the database must be accessed for every request.

In contrast, in the cookie-store mode (the default), the session is stored in the cookie as a marshaled string. This is efficient, but requires that the session be cryptographically protected for integrity; otherwise the attacker may be able to fool the server with a maliciously crafted session. Thus, in Rails, a session sent by the server is hashed with a server-side secret, and the hash is verified for every request. Unfortunately, this does not fully guarantee the integrity of sessions, because it does not guard against replay attacks. For example, consider the following code, which defines a controller method authenticate for logging in users in pubmgr.

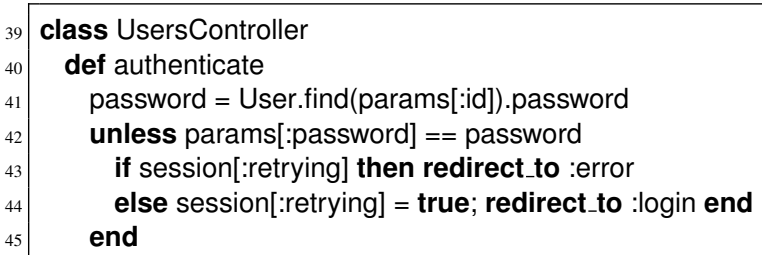




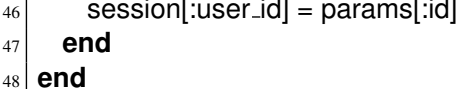

Here the user's password is looked up in the database (line 41) and, if not found, the user is given at most one chance to retry (lines 42 45). The field :retrying of the current session tracks whether the user has already retried before. Unfortunately, this code is vulnerable to a replay attack: if some user fails to $\log$ in, that user can replay the session before the try to "roll back" the state of :retrying, effectively allowing any number of tries.

The Rails documentation recommends not maintaining sensitive information in the session, although it does not help in deciding what information is sensitive. In our experience, whenever developers store non-standard information in sessions (possibly to cut down on database accesses), there is a high probability that application-specific properties can be violated using replay attacks. Safe use of cookie-store mode requires careful programming and thorough reasoning about sensitive information and sessions.

Replay attacks are easy to detect with symbolic execution, by exploring paths in which the current session may be any of a set of past sessions. In contrast, specialized techniques that do not take session replay into account would be unsound in Rails.

\subsection{Unauthorized access}

Finally, applications can implement access control to prevent unauthorized access to data and enforce specific secrecy and integrity properties. The following code snippet illustrates one way we enforce authorization in pubmgr:

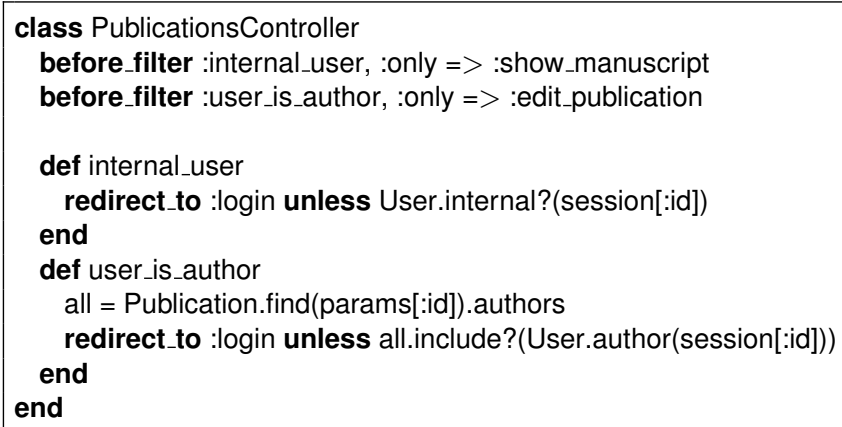

This code specifies filters before the controllers show_manuscript and edit_publication (not shown). The internal_user method checks whether the current user is a member of PLUM and, if not, redirects the user to a login controller, thus ensuring that only internal users can view unpublished manuscripts (a secrecy property). Similarly, the user_is_author method ensures that the current user is an author of the publication to be edited, so that authors can edit only their own publications (an integrity property).

As with the previous examples, we can see that Rails provides support for preventing unauthorized access, but it is still up to the programmer to determine how to code their access controls using the Rails API. Moreover, reasoning about this code relies on other security properties, such as correct password authentication and safety against session manipulation, CSRF, XSS, and so on. Our symbolic execution-based approach is very effective here because it can reason uniformly and simultaneously about all of these properties.

\section{Symbolic Execution with Rubyx}

As we saw in the previous section, ensuring that Rails's security defenses are used correctly requires reasoning about many low-level details of code. This is a perfect task for symbolic execution, which
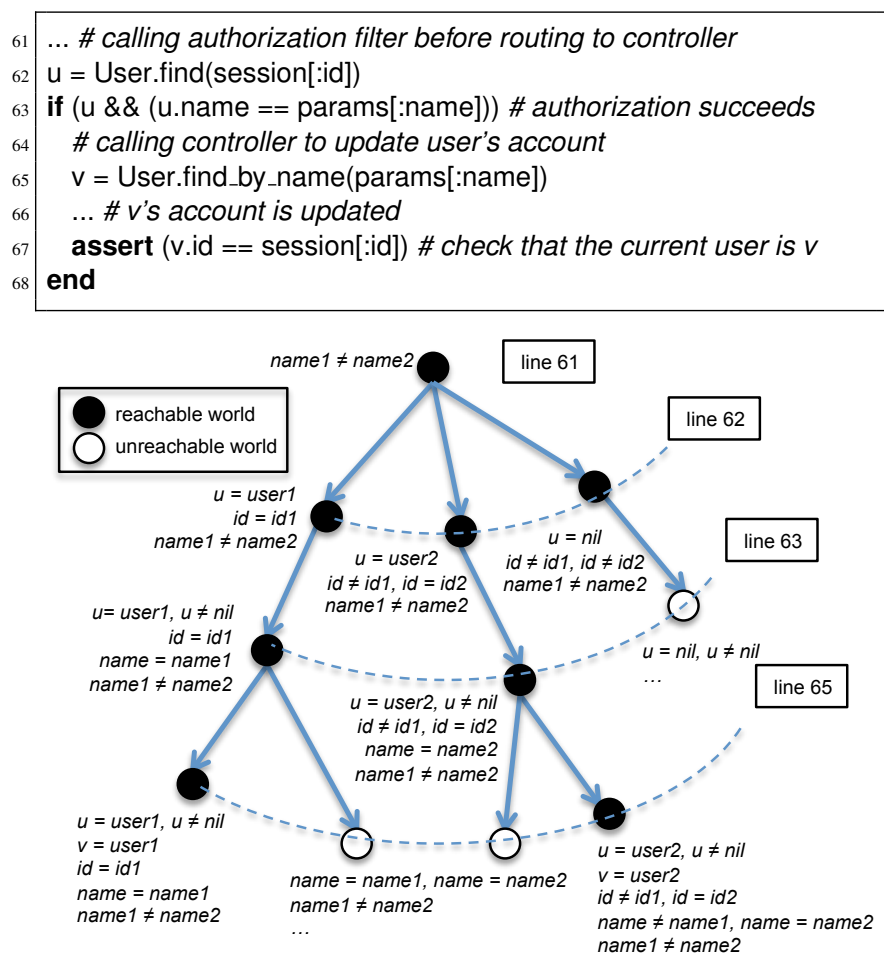

Figure 1. Partial symbolic execution tree for an example

can automatically explore many possible program executions, including corner cases that may be hard to find otherwise. In this section, we discuss the design of Rubyx, our symbolic execution engine for Rails. In Section 4, we give details of how we encode detection of security vulnerabilities using Rubyx.

At its core, Rubyx is a Ruby source code interpreter, with one key difference: in addition to modeling concrete program values, Rubyx can interpret programs that contain symbolic variables, which are unknowns that represent arbitrary sets of concrete values. Rubyx tracks these unknowns as they flow through the program. Rubyx also maintains path conditions, that track constraints on symbolic variables; initially, the path condition is simply true.

When we reach a branch with a guard $p$ that involves symbolic variables, we conceptually split the current world (i.e., the state of the Ruby program) into two new worlds, one in which $p$ is conjoined with the path condition, and one in which $\neg p$ is conjoined with the path condition. We pass the new path conditions to an SMT solver, Yices [58], to decide whether one or both conditions are actually satisfiable, i.e., whether the corresponding world is reachable from the start of the program. We continue executing the reachable world(s) forward, splitting the worlds in the future as necessary. In this way, Rubyx can simulate all paths through the program that are reachable for any concrete values that the unknown might take.

As an example, consider the code snippet at the top of Figure 1, which is taken from pubmgr (with some method calls inlined for brevity). This code does access control before updating a user's account, based on login information in the current session; it is typical of Rails applications. Using symbolic execution, we want to show that the assertion on line 67 , which checks that the user whose account is updated is currently logged in, always holds.

We start symbolic execution by sending a request that triggers the relevant controller. For this particular example, assume that when we reach line 61, session[:id] and params[:name] are symbolic variables id and name, respectively. Also assume that the 
database User contains two users, user1 and user2, whose id attributes are id1 and id2, and whose name attributes are symbolic variables name $_{1}$ and name $_{2}$, respectively. Furthermore, suppose that name $_{1} \neq$ name $_{2}$. (The application pubmgr ensures that name attributes are unique before saving users to the database.)

The bottom half of Figure 1 shows a partial graph of the worlds starting from line 61 . Reachable worlds are denoted by filled circles, and unreachable worlds are denoted by empty circles. In the initial world, the path condition is name $_{1} \neq$ name $_{2}$, from our assumptions. On line 62, we search for the currently logged-in user (from the given session id). There are three possibilities, shown in the next level of path conditions: either user1 is found; user2 is found; or no such user is found. Then on line 63, we check that the currently logged-in user's name matches the name of the user whose account will be updated. Again, there are several possible outcomes, shown next in the graph. Notice that one of the worlds on this level has a path condition containing $u=$ nil and $u \neq$ nil, which is unsatisfiable and hence this world is unreachable. This models the case when $u$ is nil after the assignment on line 62 , and hence the conjunction on line 63 is always false.

Next, on line 65 , we find the user whose name is passed as parameter to this controller, and store it in v. Again, there are several possibilities, shown in the next level of nodes in the graph. If $\mathrm{v}=$ user $i$ (for $i \in\{1,2\}$ ), then examining the path condition we can see name = name $i$ is the only possibility; the other worlds have contradictions in their path conditions. But then v.id == session[:id], again by looking at the path conditions, and hence the assertion on line 67 is always satisfied.

\subsection{Primitives for specification and verification}

Rubyx includes several built-in primitives for specifying and checking properties. The method call fresh $(n)$ returns a fresh symbolic variable named after $n$, which may be any Ruby symbol (i.e., interned Ruby string). (The name is just a convenience in understanding Rubyx's output.) Such a symbolic variable can range over any Ruby object, although its structure is constrained by subsequent operations on it. The method call assume $(p)$ conjoins the path condition with $p$, which may be any Ruby expression. (In conditional tests in Ruby, false and nil are both treated as false, and all other values are treated as true.) The assume primitive is used to specify a precondition for a property we wish to verify. Dually, the method call assert $(p)$ checks whether the path condition implies $p$; if not, Rubyx reports an error. In other words, assert specifies postconditions for properties we wish to verify.

Lastly, Rubyx supports object invariants. A method definition of the form def invariant() $p$ end in any class maintains $p$ as an invariant for all objects of that class. More precisely, we assume $p$ when an object instance is created, and we assert $p$ whenever there is an update that changes the object's state. Rubyx uses an efficient algorithm that monitors parts of the state relevant to an invariant and re-enforces the invariant only when those parts are modified.

\subsection{Integration with Yices and Optimizations}

As with most symbolic execution systems, Rubyx's capabilities and performance depend heavily on exactly how it uses Yices, its underlying SMT solver. Next, we will discuss some of the key challenges we encountered in working with Ruby, Rails, and Yices.

First, Ruby hashes, such as params and session, are used pervasively in code. To reason as generally as possible in these situations, we model hashes with uninterpreted functions in Yices, which allows us to leave the hashes as unknowns while still supporting usual lookup, update, and equality operations [49].

Second, we found that strings appear pervasively in code (in particular as inputs and outputs), and we often want to treat them as arbitrary unknowns while still supporting concatenation and other operations. In our experience, burdening Yices with constraints generated by string operations leads to poor performance. Instead, we evaluate and reason about string operations abstractly in Rubyx by maintaining partial solutions for strings in the state.

Third, defining an appropriate datatype for Ruby values in Yices is crucial for sound reasoning with (in)equalities. Unfortunately, Yices does not allow the kind of recursive datatype definitions necessary to express most Ruby values. We get around this problem by using an uninterpreted type in the definition, and carefully designing the form of constraints so that this type is always interpreted as the original datatype when solving those constraints.

Overall, we put a lot of effort into ensuring that Yices can determine the satisfiability of constraints generated by Rubyx. Our encoding guarantees Yices can prove or disprove the generated constraints, in contrast to related tools for which theorem provers may fail and require further annotations or dynamic checks [13].

Finally, we implemented a number of optimizations in Rubyx to dramatically improve performance. Most importantly, we found that many of the worlds Rubyx explores share logically identical path conditions. Thus, we maintain a cache of constraints that Yices has already solved, and avoid resolving such constraints.

Another important factor for performance is the ordering of clauses in the constraints passed to Yices. On several occasions we found that changing the ordering can reduce Yices solving time from almost an hour to less than a second. Thus, we keep constraints in a normalized form, with clauses sorted in a fixed order. Our ordering is designed to place simple conditions before more complex ones, and Yices calls have never taken more than a couple of minutes (at the extreme) with our ordering. Maintaining clauses in a normalized order also improves cache hits.

Our last important optimization is to implement some basic operations, such as lattice operations on secrecy levels (see Section 4), in Yices rather than in Rubyx. This increases the complexity of the constraints passed to Yices, but greatly reduces branching in the interpreter, which saves space and time.

\section{Analysis of Rails Applications with Rubyx}

There are three steps to analyzing Rails applications with Rubyx. First, we apply DRails, a tool we previously developed [3] to make Rails code easier to analyze by making many implicit Rails conventions explicit. For example, DRails explicitly adds database access methods to models, inserts calls to render that are implicit in Rails, and translates HTML with embedded Ruby code into pure Ruby. We likely could have used Rubyx for this purpose, but as DRails was already developed it was convenient to start with.

Second, we import proxy implementations of (a subset of) the remaining Rails API methods, the browser, and the web server. Overall, the imported code amounts to less than 150 lines of Ruby. Besides providing a functional environment for the application program to run in, the imported code specifies and verifies some common, low-level security properties.

Finally, we execute an analysis script, provided by the developer, that includes several symbolic "tests." Each such test populates the database with some symbolic objects, defines some invariants for those objects, assumes some preconditions, sends symbolic requests to the browser interface, receives responses, and asserts some postconditions. The goal of the analysis script is to direct the exploration of paths and specify and verify further, high-level properties of the application.

After running the analysis script, Rubyx reports the reachable worlds in which the properties do not hold, i.e., potential vulnerabilities detected.

Secrecy lattice and principals Low-level security properties of code ultimately rely on the preservation of secrets, such as tokens 


\begin{tabular}{l|l|}
\cline { 2 - 2 } 69 & \# No XSS \\
70 & assert (output.trust?) unless (Prin.receiver == Lattice.Bot) \\
71 & \# Secrecy \\
72 & assert (Lattice.leq (output.secrecy?, Prin.receiver)) \\
73 & \# No CSRF \\
74 & assert (Lattice.leq (Prin.receiver, Prin.sender)) if params[:post] \\
75 & assert params[:post] if (Session.modified? || Db.modified?) \\
76 & \# Authentication \\
77 & assert (Lattice.leq (session[Prin.ld], Prin.receiver)) \\
78 & assert (Lattice.leq (session[Prin.Id], Prin.sender)) if params[:post] \\
\cline { 2 - 2 } &
\end{tabular}

Figure 2. Specifications of common low-level security properties

and passwords. Thus, our specifications in step (b) are based on principals, which are secrecy levels in a lattice. In practice, the principals include various users (as identified by the web application), the web application itself $(\top)$, and the attacker $(\perp)$; they are partially ordered by their knowledge of secrets, with $T>$ honest users $>\perp$. The honest users do not know each others' secrets; dishonest users leak their secrets to the attacker; and the web application's secrets are exclusive.

Since our specifications are implemented using assume, assert, and so on in code, our proxy implementation includes a class Lattice representing secrecy levels, with constants Bot and Top and a method leq.

For any interaction (request and response) with the web application, we consider the following roles:

- $\mathcal{P}_{s}$, the principal that sent the request;

- $\mathcal{P}_{r}$, the principal that received the response;

- $\mathcal{P}_{i}$, the principal of the logged-in user

Our proxy implementation includes a class Prin to model these roles: we have $\mathcal{P}_{s}=$ Prin.sender, $\mathcal{P}_{r}=$ Prin.receiver, and $\mathcal{P}_{i}=$ session[Prin.Id]. While Prin.ld is constant for an application (e.g., for pubmgr, Prin.ld $=:$ id), the roles $\mathcal{P}_{s}, \mathcal{P}_{r}$, and $\mathcal{P}_{i}$ may change between interactions; all of these are set by the analysis script.

With these roles, we specify four low-level security propertiesNo XSS, Secrecy, No CSRF, Authentication-in the proxy browser interface, so that they are verified for every interaction. These specifications rule out the kinds of vulnerabilities discussed in Section 2. The exact specifications are shown in Figure 2; we will discuss these in detail in subsequent subsections, but for now, observe that the specifications are quite simple despite covering several different properties.

Furthermore, in combination they facilitate reasoning about end-to-end security: No XSS and Secrecy together imply the preservation of the secrecy lattice, while No CSRF and Authentication rely on the secrecy lattice to ensure the soundness of access control. Finally, other high-level properties specified in analysis scripts, such as enforcing that operations maintain consistency of the database, typically rely on these low-level properties for the specifications to be correct.

Analysis scripts Analysis scripts typically proceed as follows. First, the database is populated with objects containing unknown fields. For example, to create a User we may have:

79 User.create(fresh(:ATTRIBUTES))

The create method, automatically added by DRails, populates the fields of a new User object according to the argument and saves the object in the database. In this case, we pass in a fresh unknown hash (named after ATTRIBUTES), and so Rubyx will in turn introduce fresh unknowns for all fields in the User object. Also, those un- knowns will automatically be constrained by any invariants given by the developer. For example, we may require that the hashed_pwd attribute should be the cryptographic hash of the @password field.

Having established an appropriate state, various controllers are "tested" by sending requests with unknown parameters. For example, we may call:

${ }_{80}$ response = Browser.exec(UserController, :login, fresh(:PARAMS))

This asks the browser model to send a login request to UserController. The parameters are given by a fresh unknown hash, and so parameters like the POST/GET type of the request and the CSRF token carried by the request, as well as other request-specific parameters such as a password, will be unknowns. Any conditions on such parameters that are established dynamically by code-such as checking the type of the request, matching the CSRF token, checking the password, and so on-cause Rubyx to explore alternative worlds with the relevant constraints. And in each of those worlds, Rubyx will check the low-level security properties in Figure 2.

In addition, the developer may specify and verify other properties. For example:

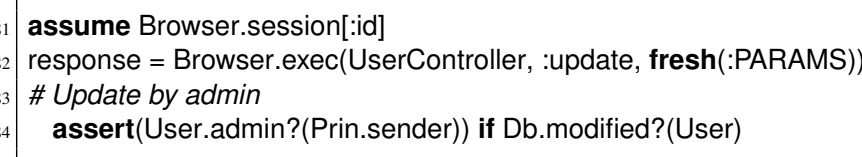

Here the developer assumes that the session id is non-nil, indicating a successful login. Then the developer sends a request for an update operation, and asserts that if the User database is modified, then the user is an administrator.

The constraint passed to Yices to check this assertion will include various facts that arise from the specifications of the lowlevel properties in Figure 2. For example, the request must be POST (by our enforcement that only POST requests may write to the database); the sender must be Browser.session[:id] (by CSRF protection and write authentication for POST requests); and Browser.session[:id] must be an admin (by a specific dynamic check in the application code, not shown).

In the following subsections, we explain our formal specifications for the common security properties in Figure 2. These specifications apply to any Rails application that uses the Rails API in standard, recommended ways to achieve security. Other, application-specific properties that may arise in analysis scripts are discussed on a case-by-case basis for our experimental benchmarks in Section 5.

\subsection{Session manipulation}

Since session manipulation can be used to violate other properties, it is important to implement sessions faithfully. Recall that in the database mode, only the session identifier is stored in a cookie, while the session information itself is stored in the database. In this mode there is not much room for session manipulation, and so in our proxy implementation, the browser is not allowed to affect the Rails internal @session field.

On the other hand, in the cookie-store mode, the browser tracks the current session internally, and sessions are stored cryptographically MAC-ed in the cookie. Thus, requests may change the current session by changing the cookie in the browser. In our proxy implementation, we abstract MAC-ing of an object $x$ by an identity operation with the side effect of including $x$ in a private list. Verifying the MAC of $x$ then reduces to checking whether $x$ is in this list. This encoding allows sessions to be replayed but not forged. Moreover, our proxy implementation ensures that principals cannot know (and thus, cannot replay) sessions received by other principals. 


\subsection{XSS}

Next we tackle XSS attacks. Recall from Section 2.1 that Rails includes two defenses: using validates_format_of to prevent fields with code from being stored in the database, or calling html_escape to sanitize strings before display.

To track sanitization, our Rails proxy implementation extends Ruby's String class with a trust level. For a string s, calling s.trust? returns true if the string is trusted, and calling s.trusted marks a string as trusted. We reduce Rails's sanitization routines into statements on trust. To encode html_escape, we simply implement it as a proxy API that returns a trusted code of the string passed to it:

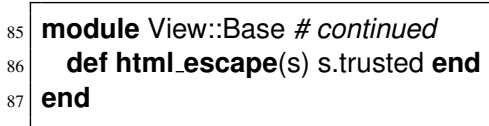

We translate validates_format_of into trust assumptions. For example, here is a translation of the call to validates_format_of from Section 2.1:

\begin{tabular}{l|l|}
88 & class Author \# continued \\
89 & def save () assume @name.trust?; Author.db $\ll$ self end \\
90 & end \\
91 & def Author.invariant ()$@ \mathrm{db}$. forall $\{\mid$ author $\mid$ author.name.trust? $\}$ end
\end{tabular}

Since we will reject any unsanitized strings, we can assume in save, which writes to the database, that name is trusted (line 89). (Note that we do not check the regular expression that name is tested against; Rubyx assumes it is correct.) This maintains an automatically generated invariant of the database: the name fields of all author objects in it are trusted (line 91). In turn, this invariant implies that any names retrieved from the database are trusted.

Once we have established the trust of strings, we can reason about XSS. In our proxy implementation, any strings displayed in a response are concatenated to the @output field of the response. In Rubyx, concatenating string $x$ with string $y$ sets the trust of $x$ to the minimum of the trusts of $x$ and $y$. We also assume that any strings passed as parameters in a request are untrusted (not shown). Then the specification of NoXSS, shown on line 70 of Figure 2, is simply that the @output field of a response must be trusted unless the output is received by an attacker.

This technique captures the key principle behind defenses against XSS: that inputs must be sanitized before they flow to outputs. By assuming that inputs may be untrusted and requiring that outputs be trusted, we effectively force inputs that flow to outputs to pass through some sanitization mechanisms, which in turn "bless" the untrusted strings as trusted.

\subsection{CSRF}

Next, we consider CSRF attacks. Recall that for any interaction with the web application, we consider principals $\mathcal{P}_{s}$ (the sender) and $\mathcal{P}_{r}$ (the receiver). CSRF is possible because the sender $\mathcal{P}_{s}$ and the receiver $\mathcal{P}_{r}$ may be different. Thus, our specification of CSRF safety is simply:

Definition 4.1 (CSRF safety). For any non-idempotent request, $\mathcal{P}_{s} \geq \mathcal{P}_{r}$.

Informally, any principal that controls behaviors of the web application must be at least as trusted the principal that views those behaviors. In particular, if $\mathcal{P}_{r}$ is an honest user, then $\mathcal{P}_{s}$ cannot be the attacker, although it may be $\mathcal{P}_{r}$ itself or another honest user $>\mathcal{P}_{r}$ (or perhaps even the web application).

We specify CSRF safety in Rubyx with two assertions. On line 74 of Figure 2, we require that POST requests have the right relationship between sender and receiver, and on line 75 we specify that any requests that change the session or database must be POST.

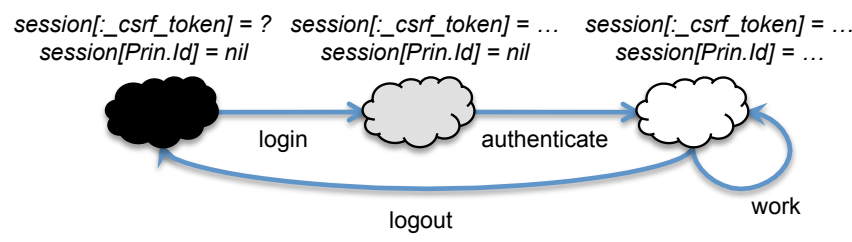

Figure 3. State machine for session

To reason about CSRF protection, we need to track the secrecy levels of various strings, so that we know which principal they came from. Hence, similarly to the trust levels in Section 4.2, we extend class String so that each string has a secrecy attribute, which returns a Lattice element.

Recall from Section 2.2 that defense against CSRF requires employing several different countermeasures: ensuring only POST requests can change state; including and checking for a secret session[:_csrf_token] in legitimate requests (achieved by calling protect_from_forgery); and calling reset_session at the right point to create fresh tokens for new users.

To understand how these three parts enforce our definition of CSRF safety, it is easiest to argue abstractly about an application. Consider Figure 3, which shows a state machine that describes the life cycle of session[:_csrf_token] and the currently logged-in principal when CSRF protection is used correctly. In this state machine, the user is initially at the black state, where session[Prin.Id] is nil and session[:_csrf_token] is irrelevant. We move to the gray state when a login request is received, at which point a fresh session[:_csrf_token] is generated. We then move to the white state when password authentication succeeds, which also sets session[Prin.ld]. We stay in that state doing work, and then eventually move back to the black state by logging out.

Thus, $\mathcal{P}_{r}$ may change only in the black state. If an implementation matches the state machine in Figure 3, then session[:_csrf_token] must change whenever $\mathcal{P}_{r}$ may have changed. More precisely, the following assertion, which we include in forgery_safe? (Section 2.2), will hold, since form_authenticity_token (Section 2.2) returns session[:_csrf_token] after generating a token if required:

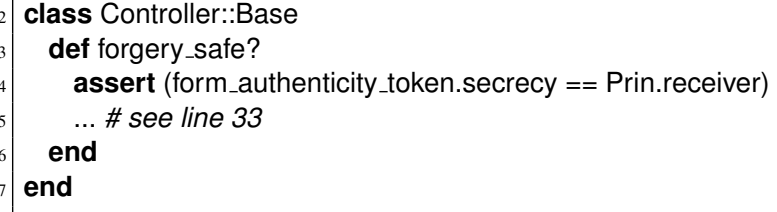

Thus we can conclude session[:_csrf_token].secrecy $=\mathcal{P}_{r}$.

Now, for any POST request, forgery_safe? (Section 2.2) establishes session[:_csrf_token] = params[:authenticity_token]. Putting these two equalities together, we have params[:authenticity_token]. secrecy $=\mathcal{P}_{r}$.

Finally, we assume that any string sent with a request must have secrecy level $\leq \mathcal{P}_{s}$ (i.e., that senders can only send messages at their secrecy level or below). Then we have params[:authenticity token].secrecy $\leq \mathcal{P}_{s}$, and thus $\mathcal{P}_{r} \leq \mathcal{P}_{s}$, which is CSRF safety for POST requests.

Note that in the state machine in Figure 3, we generate a new CSRF token for a login request. Traditionally CSRF attacks have focused only on work transitions, hence it seems we could have generated fresh tokens after authentication. However, recently login CSRF attacks have been studied [9], which focus on authentication transitions. It is easy to see that generating CSRF tokens before authentication, as we have done, prevents login CSRF attacks also.

Furthermore, note that for authentication transitions, we may reasonably assume that $P_{s} \ngtr \mathcal{P}_{r}$, i.e., $\mathcal{P}_{s}$ will never aid any less 
trusted $\mathcal{P}_{r}$ in authentication. For instance, an honest user $\mathcal{P}_{s}$ will not try to authenticate for a dishonest user $\mathcal{P}_{r}$, or for another honest user $<\mathcal{P}_{s}$. Thus, for authentication transitions that are CSRF safe we actually have $\mathcal{P}_{s}=\mathcal{P}_{r}$.

Finally, note that key to our proof of CSRF safety above was the assertion that the secrecy of the CSRF token must be equal to the receiver. We propose this as a design principle for CSRF safety. Indeed, it provides the main insight behind eliminating CSRF attacks, embodied in this combined use of protect_from_forgery and reset_session - the secrecy of the CSRF token must always be related to the principal viewing the behaviors of the web application, and since such a principal may change between logout and login, the CSRF token must also change between logout and login. Otherwise, the attacker may learn the token for an honest user, or the token for an attacker may serve an honest user-either of which can break CSRF safety.

\subsection{Authentication and Access Control}

Next, we show how Rubyx can assert the correctness of password authentication, meaning that sends and receives after authentication can be assumed to come from the logged-in user (or a more trusted principal). Formally, our specification is as follows (see lines 77 and 78 in Figure 2).

Definition 4.2 (Password authentication). Suppose that $\mathcal{P}_{i} \neq$ nil (i.e., a user is logged in).

$$
\begin{gathered}
\text { For any POST request: } \mathcal{P}_{s} \geq \mathcal{P}_{i} \\
\text { For any POST or GET request: } \mathcal{P}_{r} \geq \mathcal{P}_{i}
\end{gathered}
$$

To authenticate correctly, web applications usually store and check passwords using a combination of cryptographic hashing and "salting" to avoid known attacks. In our proxy Rails API, we include a basic model of string concatenation and hashing. Our implementation ensures that for any strings $x, y$, and $z$, if $x \neq y$ then Crypto.hash $(x) \neq \operatorname{Crypto} \cdot \operatorname{hash}(y)$ and $x+z \neq y+z$. (Here Crypto is a placeholder for the name of the relevant Ruby class.)

Given this API, Rubyx can reason about typical authentication strategies. As a concrete example, consider the following code:

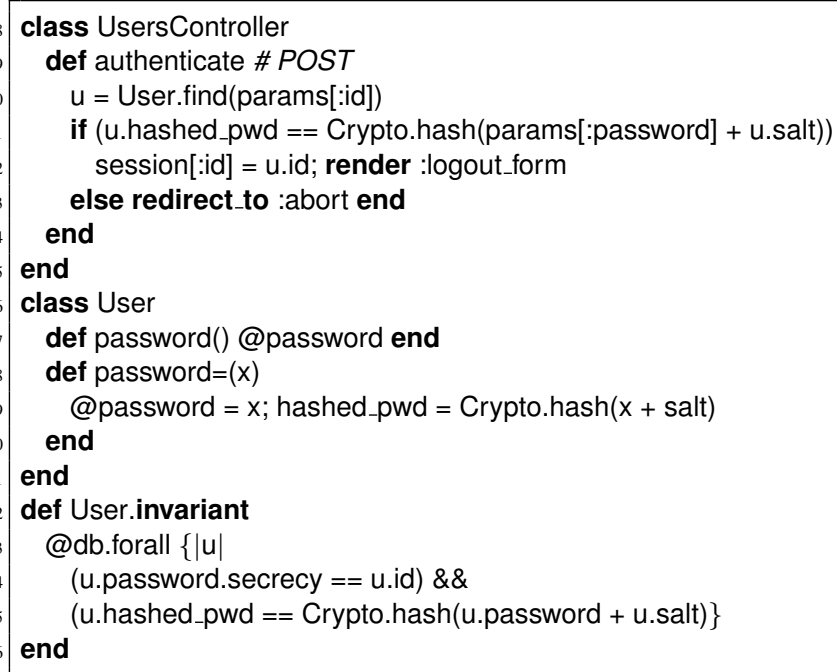

Notice that the programmer has supplied an invariant (lines 112115) that specifies that the password's secrecy level is the id of the user, as well exactly how the hashed password is computed. This invariant describes the stored password information abstractly, and lets us leave it otherwise as an unknown.

Using this invariant, Rubyx can assert that the code implements correct password authentication as follows. Suppose that $\mathcal{P}_{i} \neq$ nil.
For some user $\mathrm{u}=$ User.find (params[:id]), the code above establishes the following conditions:

$$
\begin{aligned}
\text { u.hashed_pwd } & =\text { Crypto.hash(params[:password] + u.salt) } \\
\text { u.id } & =\text { session[:id] }\left(=\mathcal{P}_{i}\right) \\
\text { u.password.secrecy } & =\text { u.id } \\
\text { u.hashed_pwd } & =\text { Crypto.hash(u.password + u.salt) }
\end{aligned}
$$

Combining these equations with those for cryptographic hashing and string concatenation above, we have params[:password].secrecy $=\mathcal{P}_{i}$. Furthermore, as in Section 4.3, we assume any string sent with a request must have secrecy level $\leq \mathcal{P}_{s}$. Then we have $\mathcal{P}_{i}=$ params[:password].secrecy $\leq \mathcal{P}_{s}$. This establishes (1). But since we are performing authentication, by CSRF safety we have $\mathcal{P}_{r}=\mathcal{P}_{s}$, and hence (2) also holds.

After authentication has occurred, $\mathcal{P}_{r}$ and $\mathcal{P}_{i}$ remain constant. (Recall that $\mathcal{P}_{r}$ can change only in the black state, and $\mathcal{P}_{i}$ does not change in the white state.) Thus $\mathcal{P}_{r} \geq \mathcal{P}_{i}$, from (2) at authentication time, continues to hold. Moreover, by CSRF safety we have $\mathcal{P}_{s} \geq \mathcal{P}_{r}$, and so (1) also continues hold.

Of course, authentication is useful only if we implement some access control, for which we need to track dynamic conditions on $\mathcal{P}_{i}$ itself, of the form $\mathcal{P}_{i} \geq \mathcal{P}_{a}$ for some access privilege $\mathcal{P}_{a}$. By the above theorem, these conditions will imply $\mathcal{P}_{s} \geq \mathcal{P}_{a}$ for write access privileges, and $\mathcal{P}_{r} \geq \mathcal{P}_{a}$ for read access privileges.

\subsection{Secrecy}

Finally, note that the above properties rely on the preservation of the secrecy lattice, i.e., principals do not eventually know secrets of unrelated principals. We specify this in line 72 of Figure 2, by requiring that any strings received in a response must have secrecy levels $\leq \mathcal{P}_{r}$, so that the receiver could have already known them.

\section{Experiments and Results}

We used Rubyx to analyze seven Rails applications with a range of non-trivial security and correctness requirements. Two applications were developed within our research group, but independently of this project (so they did not take any particular advantage of Rubyx's strengths or weaknesses). The remaining applications were obtained from external sources $[2,53]$.

\subsection{Applications and Properties}

We begin by describing each web application in our experiments, along with their application-specific access control and correctness properties. For all of the applications, we also checked for the presence of session manipulation, XSS, CSRF, and authentication attacks, using the specifications in Section 4.

As discussed earlier, the pubmgr application, which we used for examples throughout the paper, was developed by one of the authors to manage publications of our research group. In addition to the common security properties, we also checked that users are always properly authorized, as outlined in Section 2.4.

The coffee application was developed by another member of our research group to track use of a shared coffee machine. The application maintains an inventory of coffee capsules available; a count of each user's tokens, which are exchanged for coffee; and the consumption of coffee by users. Some users have administrative privileges and can refill tokens for other users and adjust the inventory of capsules. We checked to ensure that administrative privileges cannot be circumvented, and that counts of capsules, tokens, and user consumption all match up correctly.

The depot application is used as the main running example in a popular book for Rails developers [53]. Since its code is freely available, we expect that many developers use that code as a starting point for their own applications. The depot application maintains a 


\begin{tabular}{|c|c|c|c|c|c|c|}
\hline & XSS & CSRF & Auth. & Secr. & Acc. & Corr. \\
\hline pubmgr & $\sqrt{ }$ & $\times(1)$ & $\sqrt{ }$ & $\sqrt{ }$ & $\sqrt{ }$ & N/A \\
\hline coffee & $?(2)$ & $\times(1)$ & $\sqrt{ }$ & $\sqrt{ }$ & $\times(2)$ & $\sqrt{ }$ \\
\hline depot & $\times(2)$ & $\times(10)$ & $\sqrt{ }$ & $\sqrt{ }$ & & $\times^{r}(7)$ \\
\hline chuckslist & $\sqrt{ }$ & $\times(1)$ & $\sqrt{ }$ & $\sqrt{ }$ & $\times(-)$ & N/A \\
\hline boxroom & & $\times(5)$ & $\sqrt{ }$ & $\sqrt{ }$ & & $?^{r} \quad(2)$ \\
\hline mystic & $\times(17)$ & $\times(8)$ & & $\sqrt{ }$ & $\times^{r}(6)$ & N/A \\
\hline rtplan & $\sqrt{ }$ & $\times(1)$ & $\times(16)$ & $\sqrt{ }$ & $?^{r}(-)$ & $\sqrt{ }$ \\
\hline \multicolumn{2}{|c|}{$\begin{array}{l}\sqrt{ }=\text { no vuln. found } \\
(n)=n \text { fixes }\end{array}$} & \multicolumn{2}{|c|}{$\begin{array}{l}X=\text { vuln. found } \\
(-)=\text { did not fix }\end{array}$} & \multicolumn{3}{|c|}{$\begin{array}{l}?=\text { potential vuln. found } \\
r=\text { replay attack }\end{array}$} \\
\hline
\end{tabular}

Figure 4. Experimental Results and Fixes

\begin{tabular}{|r|r|rr|rr|}
\hline & LoC & \multicolumn{2}{|c|}{ Running time } & \multicolumn{2}{|c|}{ Yices stats } \\
& & orig. (s) & \% inc. & $\%$ time & \# calls \\
\hline pubmgr & 7,450 & 32.6 & $37.2 \%$ & $91.4 \%$ & 352 \\
coffee & 7,928 & 67.5 & $4.0 \%$ & $97.8 \%$ & 142 \\
depot & 5,338 & 32.5 & $16.6 \%$ & $94.8 \%$ & 265 \\
chuckslist & 12,554 & 175.2 & $10.8 \%$ & $95.7 \%$ & 519 \\
boxroom & 13,727 & 123.1 & $32.0 \%$ & $95.6 \%$ & 347 \\
mystic & 20,350 & 28.2 & $30.3 \%$ & $71.8 \%$ & 169 \\
rtplan & 9,849 & 195.3 & $1.5 \%$ & $99.1 \%$ & 163 \\
\hline
\end{tabular}

Figure 5. Performance database of products, and records orders of products by customers. It also maintains accounts of administrators, who are able to edit information on products, including their prices. We checked to ensure that administrative privileges are required to edit product information, and to introduce other administrators to the system. We also checked to ensure customers are charged the correct price for any product they order.

The chuckslist application is a classified ad system [2] inspired by craigslist. The application maintains a database of ads and their authors. It also maintains accounts for users, some of whom may have administrative privileges. Authors manage ads, users manage authors, and users with administrative privileges manage other users. The system relies on a sophisticated module to enforce access control, which we included as part of the application. We checked for several access control properties, such as: authors cannot edit ads of other authors, and administrative privileges cannot be circumvented.

The boxroom application is a secure file sharing system that maintains access control metadata for a file system [2] . Users are associated with groups, and permissions are associated with groups and files. Some of the users have administrative privileges, which are required to control these permissions. A user may access a file only if the groups they are in have permission to access that file. We checked that several important access control properties hold, including: only administrators can introduce other administrators and users into the system; non-administrators cannot modify which groups they are in or the permissions for groups and files; and users cannot access a file they do not have permission to access.

The mystic application is a trouble ticket system [2]. The application maintains a database of (outstanding and resolved) tickets, as well as accounts for customers, technical-staff members, and administrators. Customers post tickets, technical-staff members address those tickets, and administrators manage their accounts. The developers claim a clear separation of these duties in the system, and we check to ensure that this is indeed the case.

Finally, the rtplan application is a planning system for project tasks [2]. The application maintains a database of projects, each of which has several tasks. It also maintains accounts for users, who are allocated various tasks, and records the work those users have put into those tasks. Apart from expected access control properties, we checked that the project and user databases have a consistent record of the total work done.

\subsection{Attacks}

Figure 4 summarizes the results of our experiments. We group our results by property, shown across the top of the figure: No XSS, No CSRF, Authentication, Secrecy, access control, and other application-specific correctness properties. For each property, a check mark indicates no vulnerabilities found, a cross mark indicates some exploitable security vulnerability, and a question mark indicates that a potential security vulnerability exists, but it may not be exploitable. We indicate that some vulnerabilities are due to replay attacks with an $r$. For most exploitable or potential vulnerabilities, we attempted to fix the code so that the vulnerability was eliminated (as checked by Rubyx). The number of fixes is listed in the figure; we show a dash where we chose not to attempt fixes because doing so might require pervasive changes.

As we can see, Rubyx detected many vulnerabilities, and every application had at least one. At the same time, except for three cases we were able to fix the vulnerabilities with a small number of changes. Since Rubyx explores all possible program paths from a given symbolic state, once we have eliminated all detected vulnerabilities from that state we can guarantee the security of that application from any instantiation of that state.

Next, we discuss the vulnerabilities we found in more detail.

XSS Rubyx found XSS attacks in three applications. In coffee, the attributes of a capsule (including a text description) are not sanitized, and hence can be used for XSS attacks. We categorized this issue as a potential, rather than definite, vulnerability because only administrators can modify attributes. However, this still reflects poor security practice because administrators could use XSS attacks to steal passwords from other users.

In depot, a product's information includes information on orders that were placed on it, and the text of such orders is not sanitized. Thus, any customer that places an order can mount XSS attacks on other customers and administrators who view this data. Similarly, in mystic, users can mount XSS attacks on other users.

CSRF Rubyx found CSRF attacks against all applications. In pubmgr, the vulnerability exists because the controller for logging in a new user does not use reset_session-instead it simply resets session[:user] to nil, but does not also set session[:_csrf_token] to nil. In coffee, the developer does use reset_session, but in the controller for logging out a user instead of logging in-hence CSRF attacks are enabled by simply not logging out. Similar problems occur in chuckslist, rtplan, depot, boxroom, and mystic. Moreover, the latter three also do not require that all state-changing requests are POST, enabling yet more CSRF attacks.

Authentication Only one application, rtplan, failed to correctly authenticate users. Strangely, here users do not require passwords to log in, although users do have password attributes in the model.

Access control Rubyx found critical violations of access control in several applications. In coffee, non-administrators can change their token counts or even grant themselves administrator privileges. The problem is that the code to update a user's information can write to all of the attributes of that user, even in cases when only some information should being updated. The developer of coffee tried to prevent the token count and administrator bit from being updated in non-administrative mode by rendering one of them as read-only and not rendering the other in the relevant form. Unfortunately, this is not enough: a simple browser setting can make read-only parameters writable, and any missing parameters can be passed by extending the form manually. This is an interesting ex- 
ample, because it shows how even a developer who pays attention to security can get it wrong.

In chuckslist, administrative privileges are not protectedanybody can sign up as an administrator, and any existing user can obtain administrative privileges. Additionally, any author can trivially become a user and manage other authors in the system. For example, an author and all its ads may be removed without an existing user logging in. Finally, any author can edit any ad in the system. In fact, chucklist does not actually maintain any relationship between users and authors, which is surprising, because at the database level they have very similar attributes.

In mystic, the claimed separation of duties is violated. Anybody can sign up as an administrator and can modify others' accounts. Furthermore, privileges granted to a logged-in user are stored in the session, and so session replay can be used to defeat revocation. For example, even if a technical-staff member's privileges are revoked by an administrator, it may replay a stale session to keep viewing information on outstanding tickets.

In rtplan, any user can promote itself or anyone else to an administrator. Furthermore, the isadmin attribute of a user is stored in the session, so a user whose administrative privileges have been revoked can keep acting as an administrator by replaying a stale session. Strangely, however, administrator privileges do not seem to have any function in this application, so we must consider these attacks benign.

Application-specific security properties Finally, Rubyx found violations of application-specific correctness properties in two cases. In depot, Rubyx found attacks in which the price in an order does not match the price of the ordered products. The problem is that the shopping cart of a customer-which is used to place the order-is stored in the session, which can be replayed. Thus, a customer may add his favorite products to the cart, save the session, and empty the cart. Later, the administrator may increase the prices of those products. But then the customer can replay his session and check out, paying the lower prices. Indeed, the database is not consulted before placing an order-there is complete trust in the session.

In boxroom, Rubyx found a potential vulnerability that is currently not exploitable (but may be in the future). The problem is that a clipboard, containing metadata of files, is stored in the sessionand, as above, that metadata may not be in sync with the database if the session is replayed. Thus, a user may be able to "save" files in the clipboard and (much later) "copy" them back into the file system, thereby restoring any access control metadata associated with those files-even if such metadata was modified by an administrator in the interim. However, while there is a controller for copying files from the clipboard to the file system, it is currently a stub that does nothing. Thus, this attack is latent until that controller is implemented.

\subsection{Performance}

Finally, we briefly discuss Rubyx's performance, summarized in Figure 5. The applications we analyzed ranged from $5 \mathrm{k}$ to $20 \mathrm{k}$ lines of code. The running times for these applications (with fixes, where relevant) were between half a minute to 3 minutes. On average, around $90 \%$ of the time was spent on calls to Yices; indeed, one such call took 75 seconds out of 195 seconds of total running time. Caching such calls not only improved performance (we observed a high cache hit rate), but also allowed us to analyze the applications "incrementally." Indeed, during our experiments we often ran partial analysis scripts on an unfamiliar application to get intermediate results, and then incrementally added assumptions and assertions to get the final results; caching prevented the cumulative analysis times from blowing up quadratically across runs. The resulting experience was much like testing. To measure this effect, note that re-running the analyses took only between $2-40 \%$ of the original running times, and these time fractions went down as the time fractions spent on calls to Yices went up.

\section{Related Work}

Some necessary background on important threats and defenses for web-application security can be found in [51, 64], while an excellent resource for Rails security in particular is [65].

Much research on web-application security has focused on settings where applications are untrusted, and users must be protected from applications. Thus, client-side defenses have been studied $[36,41,55]$, and there has been important progress on browser security $[4,10,29,52,69]$, and on JavaScript security in particular $[4,30,44,45,55,69]$. In contrast, applications are not considered inherently malicious in our setting - we assume that some users may be malicious, and we care about verifying that applications and other users are protected from such malicious users.

The need for end-to-end web application security has been previously argued [26]. Existing techniques for achieving end-toend security for web applications include type systems [11, 19], logics [12], and secure compilation [7, 20, 21, 61]. Most of these techniques rely on lattice-based security with labels [23, 25, 46], as we also do. An important difference between such techniques and ours is that we require far less annotations.

Many specialized techniques have been developed to analyze particular security properties of web applications. Some of these focus on statically typed languages such as Java [31, 43, 48, 60]. Static security analysis techniques have also been proposed for scripting languages such as PHP [38, 63, 66]. However, such techniques are often too imprecise in such a setting. Thus, most other techniques focus on dynamic security analysis, including testing and monitoring [22, 24, 28, 31, 34, 55, 57, 68]. Finally, several techniques combine static and dynamic analysis for security $[8,32,35$, $42,62]$. Our symbolic execution-based technique is similar in the spirit, since it exploits the precision of dynamic analysis for guarantees similar to static analysis. Some recent tools use symbolic execution for security analysis of JavaScript programs $[5,54]$.

Very few tools are designed for programmability, which allows analysis of a wide variety of common properties as well as application-specific properties, such as ours. Research on "extended security checking" explores this approach [18, 48, 56, 67].

Finally, most of the existing work on understanding and analyzing web application security focuses on injection attacks [59]. In comparison, the other kinds of attacks we consider in this paper have received far less attention; notable exceptions include [24, 50] on access control and authentication, [37] on session integrity, and $[9,47]$ on CSRF. Some recent work studies new kinds of attacks that we do not consider in this paper; they include clickjacking [6] and XCS (cross-channel scripting) [15] in particular.

\section{Conclusion}

We described a new approach that uses symbolic execution to reason about the security of Ruby-on-Rails web applications. Our symbolic executor, Rubyx, uses a simple assume/assert language that can describe a wide range of properties. We discussed XSS, CSRF, session manipulation, and unauthorized access in Ruby. We showed how to specify protection against these attacks in Rubyx, using basic notions such as principals, secrecy, and trust levels. We used Rubyx to check seven applications against our specifications, and found a wide variety of vulnerabilities. In particular, we observed that developers often do not appreciate defenses against session replay and CSRF attacks. Overall, our results suggest that symbolic execution is a promising approach for analyzing web applications for security vulnerabilities. 


\section{References}

[1] Ruby. http://www.ruby-lang.org/en/.

[2] Rubyforge. http://rubyforge.org/.

[3] Jong-hoon An, Avik Chaudhuri, and Jeffrey S. Foster. Static typing for Ruby on Rails. In ASE '09: Proceedings of the 2009 IEEE/ACM International Conference on Automated Software Engineering, pages 590-594, Washington, DC, USA, 2009. IEEE Computer Society.

[4] V. Anupam and A. Mayer. Security of web browser scripting languages: Vulnerabilities, attacks, and remedies. In Proceedings of the 7th USENIX Security Symposium, pages 187-200, 1998.

[5] S. Artzi, A. Kiezun, J. Dolby, F. Tip, D. Dig, A. Paradkar, and M.D. Ernst. Finding bugs in web applications using dynamic test generation and explicit state model checking. IEEE Transactions on Software Engineering, 2010.

[6] M. Balduzzi, M. Egele, E. Kirda, D. Balzarotti, and C. Kruegel. A Solution for the Automated Detection of Clickjacking Attacks. In ASIACCS, 2010.

[7] I.G. Baltopoulos and A.D. Gordon. Secure compilation of a multitier web language. In Proceedings of the 4th international workshop on Types in language design and implementation, pages 27-38. ACM New York, NY, USA, 2009.

[8] D. Balzarotti, M. Cova, V. Felmetsger, N. Jovanovic, E. Kirda, C. Kruegel, and G. Vigna. Saner: Composing static and dynamic analysis to validate sanitization in web applications. In IEEE Symposium on Security and Privacy, volume 66. Citeseer, 2008.

[9] A. Barth, C. Jackson, and J.C. Mitchell. Robust defenses for crosssite request forgery. In Proceedings of the 15th ACM conference on Computer and communications security, pages 75-88. ACM, 2008.

[10] A. Barth, C. Jackson, and C. Reis. The Google Chrome Team. The Security Architecture of the Chromium Browser. WWW2009, April, 2009 .

[11] Jesper Bengtson, Karthikeyan Bhargavan, Cédric Fournet, Andrew D. Gordon, and Sergio Maffeis. Refinement types for secure implementations. In CSF '08: Proceedings of the 2008 21 st IEEE Computer Security Foundations Symposium, pages 17-32, Washington, DC, USA, 2008. IEEE Computer Society.

[12] Karthikeyan Bhargavan, Cédric Fournet, and Andrew D. Gordon. Verifying policy-based security for web services. In CCS '04: Proceedings of the 11th ACM conference on Computer and communications security, pages 268-277, New York, NY, USA, 2004. ACM.

[13] Gavin M. Bierman, Andrew D. Gordon, Cătălin Hrițcu, and David Langworthy. Semantic subtyping with an SMT solver. Preliminary report available at http://research.microsoft.com/enus/um/people/adg/Publications/dminor.pdf.

[14] Prithvi Bisht, P. Madhusudan, and V. N. Venkatakrishnan. Candid Dynamic candidate evaluations for automatic prevention of SQL injection attacks. ACM Trans. Inf. Syst. Secur., 13(2):1-39, 2010.

[15] H. Bojinov, E. Bursztein, and D. Boneh. XCS: cross channel scripting and its impact on web applications. In Proceedings of the 16th ACM conference on Computer and communications security, pages 420431. ACM, 2009

[16] C. Cadar, D. Dunbar, and D. Engler. Klee: Unassisted and automatic generation of high-coverage tests for complex systems programs. In USENIX Symposium on Operating Systems Design and Implementation (OSDI), 2008

[17] C. Cadar, V. Ganesh, P. Pawlowski, D. Dill, and D. Engler. EXE: A system for automatically generating inputs of death using symbolic execution. In Proceedings of the 13th ACM Conference on Computer and Communications Security (CCS). Citeseer, 2006.

[18] Brian V. Chess. Improving Computer Security using Extended Static Checking. In IEEE Symposium on Security and Privacy, pages 160$176,2002$.

[19] Adam Chlipala. Ur: Statically-typed metaprogramming with typelevel record computation. In Proceedings of the ACM SIGPLAN 2010 Conference on Programming Language Design and Implementation (PLDI'10), 2010.
[20] S. Chong, J. Liu, A.C. Myers, X. Qi, K. Vikram, L. Zheng, and $\mathrm{X}$. Zheng. Building secure web applications with automatic partitioning. Communications of the ACM, 52(2):79-87, 2009.

[21] S. Chong, K. Vikram, A.C. Myers, et al. SIF: Enforcing confidentiality and integrity in web applications. In Proc. 16th USENIX Security, 2007.

[22] James Clause, Wanchun Li, and Alessandro Orso. Dytan: a generic dynamic taint analysis framework. In ISSTA '07: Proceedings of the 2007 international symposium on Software testing and analysis, pages 196-206, New York, NY, USA, 2007. ACM.

[23] Brian J. Corcoran, Nikhil Swamy, and Michael Hicks. Cross-tier, label-based security enforcement for web applications. In SIGMOD '09: Proceedings of the 35th SIGMOD international conference on Management of data, pages 269-282, New York, NY, USA, 2009. ACM.

[24] M. Dalton, C. Kozyrakis, and N. Zeldovich. Nemesis: Preventing Authentication \& Access Control Vulnerabilities in Web Applications. In Proceedings of the 18th Annual USENIX Security Symposium, 2009.

[25] Dorothy E. Denning. A Lattice Model of Secure Information Flow. Communications of the ACM, 19(5):236-243, May 1976.

[26] U. Erlingsson, B. Livshits, and Y. Xie. End-to-end web application security. In Proceedings of the Workshop on Hot Topics in Operating Systems, 2007.

[27] Cédric Fournet, Andrew D. Gordon, and Sergio Maffeis. A type discipline for authorization in distributed systems. Proc. of CSF'07, 2007.

[28] A. Futoransky, E. Gutesman, and A. Waissbein. A dynamic technique for enhancing the security and privacy of web applications. Proc. Black Hat USA, 2007.

[29] T. Groß, B. Pfitzmann, and A.R. Sadeghi. Browser model for security analysis of browser-based protocols. Computer Security-ESORICS 2005, pages 489-508, 2005.

[30] Arjun Guha, Shriram Krishnamurthi, and Trevor Jim. Using static analysis for Ajax intrusion detection. In $W W W, 2009$.

[31] V. Haldar, D. Chandra, and M. Franz. Dynamic taint propagation for Java. In Proceedings of the 21st Annual Computer Security Applications Conference, pages 303-311. Citeseer, 2005.

[32] William G. J. Halfond and Alessandro Orso. Amnesia: analysis and monitoring for neutralizing SQL-injection attacks. In ASE '05: Proceedings of the 20th IEEE/ACM international Conference on Automated software engineering, pages 174-183, New York, NY, USA, 2005. ACM.

[33] Kelly Jackson Higgins. CSRF vulnerability: A 'sleeping giant'. United Business Media via http://www.darkreading.com/security/appsecurity/showArticle.jhtml?articleID=208804131.

[34] Yao-Wen Huang, Shih-Kun Huang, Tsung-Po Lin, and Chung-Hung Tsai. Web application security assessment by fault injection and behavior monitoring. In $W W W$ '03: Proceedings of the 12th international conference on World Wide Web, pages 148-159, New York, NY, USA, 2003. ACM.

[35] Yao-Wen Huang, Fang Yu, Christian Hang, Chung-Hung Tsai, DerTsai Lee, and Sy-Yen Kuo. Securing web application code by static analysis and runtime protection. In $W W W$, pages 40-52, 2004.

[36] T. Jim, N. Swamy, and M. Hicks. Defeating script injection attacks with browser-enforced embedded policies. In Proceedings of the 16th international conference on World Wide Web, page 610. ACM, 2007.

[37] M. Johns. SessionSafe: Implementing XSS immune session handling. Computer Security-ESORICS 2006, pages 444-460, 2006.

[38] N. Jovanovic, C. Kruegel, and E. Kirda. Pixy: A static analysis tool for detecting web application vulnerabilities. In 2006 IEEE Symposium on Security and Privacy, page 6, 2006.

[39] Y.P. Khoo, B.Y.E. Chang, and J.S. Foster. Mixing Type Checking and Symbolic Execution. In PLDI, 2010.

[40] James C. King. Symbolic execution and program testing. Commun. ACM, 19(7):385-394, 1976. 
[41] Engin Kirda, Christopher Kruegel, Giovanni Vigna, and Nenad Jovanovic. Noxes: a client-side solution for mitigating cross-site scripting attacks. In SAC '06: Proceedings of the 2006 ACM symposium on Applied computing, pages 330-337, New York, NY, USA, 2006. ACM.

[42] Monica S. Lam, Michael Martin, Benjamin Livshits, and John Whaley. Securing web applications with static and dynamic information flow tracking. In PEPM, pages 3-12, 2008.

[43] V.B. Livshits and M.S. Lam. Finding security vulnerabilities in Java applications with static analysis. In USENIX Security, 2005.

[44] S. Maffeis, J.C. Mitchell, and A. Taly. Object capabilities and isolation of untrusted web applications. In Proc of IEEE Security and Privacy'10. IEEE, 2010.

[45] S. Maffeis and A. Taly. Language-based isolation of untrusted Javascript. In $C S F, 2009$.

[46] J. Magazinius, A. Askarov, and A. Sabelfeld. A Lattice-based Approach to Mashup Security. In ASIACCS, 2010.

[47] Z. Mao, N. Li, and I. Molloy. Defeating Cross-Site Request Forgery Attacks with Browser-Enforced Authenticity Protection. Financial Cryptography and Data Security, pages 238-255, 2009.

[48] M. Martin, B. Livshits, and M.S. Lam. Finding application errors and security flaws using PQL: a program query language. ACM SIGPLAN Notices, 40(10):383, 2005.

[49] J. McCarthy. Towards a mathematical science of computation. Information Processing, 62:21-28, 1962.

[50] G. Naumovich and P. Centonze. Static analysis of role-based access control in J2EE applications. ACM SIGSOFT Software Engineering Notes, 29(5):1-10, 2004.

[51] OWASP. The ten most critical web application risks, 2010. http://www.owasp.org/images/0/0f/OWASP_T10_-_2010_rc1.pdf.

[52] B. Ross, C. Jackson, N. Miyake, D. Boneh, and J.C. Mitchell. Stronger password authentication using browser extensions. In USENIX Security, 2005.

[53] Sam Ruby, Dave Thomas, and David Heinemeier Hansson. Agile Web Development with Rails. The Pragmatic Bookshelf, 2009.

[54] P. Saxena, D. Akhawe, S. Hanna, F. Mao, S. McCamant, and D. Song. A Symbolic Execution Framework for JavaScript, 2010.

[55] P. Saxena, S. Hanna, P. Poosankam, and D. Song. FLAX: systematic discovery of client-side validation vulnerabilities in rich web applications. In Proceedings of the Network and Distributed System Security Symposium, 2010.

[56] David Scott and Richard Sharp. Abstracting application-level web security. In $W W W$ '02: Proceedings of the 11th international conference on World Wide Web, pages 396-407, New York, NY, USA, 2002. ACM.

[57] R. Sekar. An efficient black-box technique for defeating web application attacks. In Network and Distributed Systems Symposium (NDSS 2009), 2009.

[58] SRI. Yices: An SMT solver. http://yices.csl.sri.com/.

[59] Zhendong Su and Gary Wassermann. The essence of command injection attacks in web applications. In POPL '06: Conference record of the 33rd ACM SIGPLAN-SIGACT symposium on Principles of programming languages, pages 372-382, New York, NY, USA, 2006. ACM.

[60] Omer Tripp, Marco Pistoia, Stephen J. Fink, Manu Sridharan, and Omri Weisman. TAJ: Effective taint analysis for Java. In PLDI, 2009.

[61] K. Vikram, A. Prateek, and B. Livshits. Ripley: automatically securing web 2.0 applications through replicated execution. In Proceedings of the 16th ACM conference on Computer and communications security, pages 173-186. ACM, 2009.

[62] P. Vogt, F. Nentwich, N. Jovanovic, E. Kirda, C. Kruegel, and G. Vigna. Cross-site scripting prevention with dynamic data tainting and static analysis. In Proceeding of the Network and Distributed System Security Symposium (NDSS07). Citeseer, 2007.

[63] Gary Wassermann and Zhendong Su. Sound and precise analysis of web applications for injection vulnerabilities. In PLDI '07: Proceed- ings of the 2007 ACM SIGPLAN conference on Programming language design and implementation, pages 32-41, New York, NY, USA, 2007. ACM.

[64] Web Application Security Consortium. Web application security statistics, 2008. http://projects.webappsec.org/Web-ApplicationSecurity-Statistics.

[65] Heiko Webers. Ruby on rails security, v2. OWASP report available at http://www.owasp.org/images/2/26/Owasp-rails-security.pdf.

[66] Y. Xie and A. Aiken. Static detection of security vulnerabilities in scripting languages. In USENIX Security, pages 179-192, 2006.

[67] Alexander Yip, Xi Wang, Nickolai Zeldovich, and M. Frans Kaashoek. Improving application security with data flow assertions. In SOSP '09: Proceedings of the ACM SIGOPS 22nd symposium on Operating systems principles, pages 291-304, New York, NY, USA, 2009. ACM.

[68] S. Yoshihama, T. Yoshizawa, Y. Watanabe, M. Kudoh, and K. Oyanagi. Dynamic information flow control architecture for web applications. Computer Security-ESORICS 2007, pages 267-282, 2007.

[69] Dachuan Yu, Ajay Chander, Nayeem Islam, and Igor Serikov. Javascript instrumentation for browser security. In POPL '07: Proceedings of the 34th annual ACM SIGPLAN-SIGACT symposium on Principles of programming languages, pages 237-249, New York, NY, USA, 2007. ACM. 